\title{
FORMACIÓN DE JÓVENES CIENTÍFICOS
}

\author{
Raúl Ishiyama Cervantes ${ }^{1}$, Dilma Diany Hallasi Roselló ${ }^{2}$
}

\section{RESUMEN}

Constantemente se habla de la importancia de la investigación científica para el desarrollo del país. No hay uniformidad en la enseñanza de diseñar el proyecto, en el desarrollo de la investigación ni para la publicación. En La Universidad Peruana Cayetano Heredia durante 25 años los alumnos de los primeros años aprendieron a investigar en forma práctica. La falta de infraestructura, de materiales y de equipos no fue impedimento. Al inicio del curso se explico la importancia de la investigación. Aprendieron a usar libros y revistas para la búsqueda de información sobre temas similares. La metodología fue simple así como el diseño del proyecto y la presentación del resultado de la investigación. Para escribir el informe contaron con "Pautas para al publicación de tesis". A fines del semestre, los alumnos expusieron sus trabajos ante sus compañeros en la forma como se hace en los congresos científicos. El objetivo fue atraerlos hacia la investigación, en forma practica y entretenida que les dio satisfacciones personales. Esta experiencia está a disposición de los interesados.

Palabras clave: Investigación, universitario, proyecto, desarrollo, publicación.

\section{ABSTRACT}

Constantly it is spoken of the importance of the scientific research for the development of the country. There is no uniformity in teaching to design the project, in the development of the investigation neither of the publication. In the Cayetano Heredia Peruvian University during 25 years the students of the first years learned to investigate in practical form. The lack of infrastructure, materials and equipment was not impediment. At the beginning of the course, I explain the importance of the investigation. They learned to use books and magazines for searching information on similar subjects. The methodology was simple as well as the design of the project and the presentation of the result of the investigation. To write the report they counted on "Steps for publishing the thesis". By the end of the semester, the students exposed their works to their companions in similar form as the scientific congresses. The objective was to attract them towards the investigation, in practical and entertained form that gave to them personal satisfactions. This experience is to disposition of the interested ones.

Key words: Investigation, college student, project, development, publication.

\section{INTRODUCCIÓN}

Se habla de la importancia de la investigación científica para el desarrollo del país; pero no se aplica la forma universal de enseñar como se diseña el proyecto, como se realiza ni como se publica la investigación. Las universidades del país por su autonomía tienen su propia forma de reglamentar la investigación así como la tesis. Tampoco existe uniformidad de criterio entre los asesores por lo que el joven interesado en incursionar en investigar se siente confundido más que estimulado. La solución es uniformizar criterios, tarea que están realizando el CONCYTEC y la Oficina de Promoción de la Investigación de la Universidad Peruana Cayetano Heredia. EI CONCYTEC desde hace 15 años está incentivando la investigación científica desde la escuela con la Feria Escolar Nacional de Ciencia y Tecnología (FENCYt), es el semillero de los futuros investigadores; la continuidad esta en la Feria Universitaria $(1,2)$.

Los cursos de investigación que se imparten en las universidades son complejos, incluye numerosos requisitos, lo que hace que los proyectos demoren semanas en ser estructurados y meses en ser aprobados. Cuando se trata de desarrollarlo se demora mucho por ser complejo. Obligado por el compromiso el autor presenta un informe mediocre, muy pocas veces en forma de artículo publicable.

¿Para qué se necesita hacer un proyecto perfecto? Es un proyecto, por lo tanto modificable. Al desarrollarlo se presentan cambios. La innovación con base a la investigación nunca es una línea recta que va directamente del problema a la solución, de ser así casi todo se habría solucionado de inmediato.

Una cualidad humana es la curiosidad, desde que el individuo nace está indagando lo que acontece a su alrededor, cuando el niño asiste a la escuela se encuentra con numerosas reglas que debe cumplir, lo que va atenuando su interés por averiguar lo que llama su atención, se convierte en un elemento que escucha y escribe lo que oye, no tiene oportunidad de discutir los asuntos de su interés. Cuando es mayor y quiere dedicarse a investigar en la universidad se ve obligado a seguir cursos complicados para aprender la "metodología" de la investigación (3)..

Pudo haber aprendido naturalmente con la práctica si se le hubiera dejado en libertad para trabajar sus ideas desde los primeros años de escolaridad, siguiendo el método del aprendizaje por la investigación como lo

\footnotetext{
1 Doctor en Ciencias Biológicas. Profesor Extraordinario Investigador de la Facultad de Ciencias y Filosofia; miembro de la Oficina de Promoción de la Investigación de la Universidad Peruana Cayetano Heredia. Asesor de la Feria Escolar y Universitaria, y miembro del Comité Nacional para la Edición de Publicaciones Científicas del CONCYTEC. Asesor de tesis y trabajos de investigación. E-mail: rishiyama@hotmail.com

2 Medico Cirujano. Centro de Estudios e Investigación en Medicina de Altura (CEIMA) Puno. Jefa de la Brigada ELITES de Sucre, Ayacucho, Dirección Regional de salud de Ayacucho (DIRESA) E-mail: dhallasi@hotmail.com
} 
hacen quienes participan en la FENCYT (4).

En la Universidad Peruana Cayetano Heredia desde 1971 y por 24 años consecutivos los alumnos del segundo año de estudios generales aprendieron la investigación en forma práctica, produciendo 700 trabajos que se dejaron en la biblioteca central. No se dictó ningún curso de metodología de la investigación, la idea fue atraerlos hacia esta actividad proporcionándole pautas sencillas, mostrando que es un trabajo entretenido que brinda satisfacciones personales.

La motivación fue el estímulo de realizar el trabajo de investigación, por esa época decían que solo podían hacerlo los investigadores calificados. Los resultados que obtuvieron trabajando en forma similar a los investigadores aumentó su autoestima sumado a la satisfacción de presentar el fruto de su constancia en congresos de jóvenes científicos y de profesionales. Varios de estos trabajos se convirtieron en tesis de grado.

\section{MATERIAL Y MÉTODOS}

Ante la falta de infraestructura, materiales y equipos, se optó que aplicaran las pautas básicas que se les brindó al inicio del curso y cómo buscar temas originales, preparar el proyecto y desarrollarlo con la ayuda de un asesor que fue un profesor $o$ un científico de la universidad o de otra institución científica. Para realizar el trabajo se formaron grupos de tres alumnos como máximo. El lugar de la investigación fue de libre elección de cada grupo, muchos investigadores de la Universidad brindaron espacio en sus laboratorios.

Al inicio del curso se explicó la importancia de la investigación, en que consiste, como se realiza y como se publica. Aprendieron a usar los libros y revistas para la rápida búsqueda bibliográfica de temas similares. Se insistió en el uso del Sistema Internacional de Unidades. La evaluación se realizó con base a la presentación de las tareas cronogramadas que figuraba en el syllabus. Si el grupo no cumplía el mínimo exigido o no exponía el trabajo quedaba desaprobado y en el siguiente semestre debía planificar un nuevo tema.

En los últimos 15 años, bajo la dirección del CONCYTEC, se desarrolla la
FENCYT en la que participan más de cuatro millones de estudiantes en el ámbito de colegio de todo el país. Las indicaciones para realizarlas están en la "Guía del Participante", redactada en forma muy sencilla si se compara con los cursos universitarios.

\section{RESULTADOS}

El procedimiento sirvió para demostrar que la simplicidad natural, tanto del proyecto, como el desarrollo de la investigación y la presentación de los resultados es la mejor forma de incentivar a los jóvenes para dedicarse a la investigación. Los alumnos aprendieron a conocer la estructura y el manejo de las revistas científicas en todas sus modalidades para obtener información para el diseño de su proyecto, desarrollar su investigación, escribir el informe en formato de tesis y exponerlo en una actividad similar a un congreso científico. Muchos de los trabajos fueron perfeccionados y al finalizar los estudios fueron presentados como tesis por sus autores.

Mientras duró el curso entre 1971 y 1995 los estudiantes hicieron 700 trabajos que se encuentran en la biblioteca de la Universidad, algunos han desaparecido, probablemente ampliado se han convertido en tesis de otras personas. Los jóvenes aprendieron que la investigación es divertida para la mayoría, otros, los menos, descubrieron que no tenían vocación por la investigación, comprobada con su experiencia personal en forma real.

De acuerdo al cronograma pre establecido se llevó un control sobre el avance de la investigación, en cada oportunidad la libreta de anotaciones debía tener la fecha de la última verificación con la firma del asesor.

Para escribir el informe final tuvieron "Pautas para la publicación de Tesis", este trabajo modificado se llama ahora "Pautas para la publicación de artículos científicos". Citando las referencias bibliográficas en forma correcta porque estas avalan en gran parte la seriedad de la investigación.

A fines del semestre, en horas de clase los alumnos expusieron sus trabajos ante sus compañeros y personas interesadas en la forma como se hace en los congresos científicos.

\section{DISCUSIÓN}

La falta de infraestructura no fue impedimento para desarrollar la investigación, la mayoría de los científicos de la universidad proporcionaron las facilidades de sus laboratorios y los asesoraron; algunos lo hicieron en otros lugares, siempre bajo la supervisión de un asesor. El asesor que no fue de la universidad se comprometió por escrito cumplir con el compromiso, ninguno falló por su seriedad profesional $(5,6)$..

Fue una experiencia original, se indagó si existía un curso igual en los países latinoamericanos, no se encontró información de algo similar. Los cursos de metodología de la investigación que se dictaban y aún se hacen son eminentemente teóricos, con innumerables clasificaciones y gran cantidad de definiciones que en lugar de ayudar a estimular el interés del joven, complica y angustia al futuro investigador, porque además tenía que rendir exámenes.

Cuando se aplicó esta metodología, no se pretendió que todos fueran investigadores, no todos tienen las cualidades; lo que interesó fue descubrir las potencialidades como investigador, comprobado con hechos.

Por razones que no viene al caso explicar, ese proceso se interrumpió después de 24 años continuados. Esta experiencia, con modificaciones actualizadas está a disposición de los interesados, de tal manera que no tengan que realizar un nuevo ensayo.

Los cursos de investigación científica - metodología de la investigación está constituido por una serie de aspectos complicados que se apartan de la investigación natural. En los últimos años se han creado una serie de terminologías y clasificaciones que puede ser útiles en determinados casos, pero ajenos a lo natural de como lo realizaron los investigadores clásicos.

Hay que ir más allá de lo conocido depurando la mente de prejuicios. A veces lo que conocemos es el mayor obstáculo que lo que no conocemos, tenemos arraigado los prejuicios en algunos casos en forma de reglas. Con pensamiento abierto necesitamos ayuda para salir y mantenernos fuera de los surcos que han dejado esas reglas $(7,8)$. 
La enseñanza para realizar la investigación debe ser atractiva, de tal manera que el joven lo tome como algo natural, interesante que satisfaga su curiosidad para obtener satisfacción personal y contribuir con el desarrollo de la sociedad.

\section{REFERENCIAS BIBLIOGRÁFICAS}

1. Ishiyama Cervantes, Raúl. Las actividades científicas extraescolares para el aprendizaje como investigación. Lima: Ministerio de Educación, CONCYTEC; 1997

2. Ishiyama Cervantes Raúl. Editor. Feria escolar Nacional de ciencia y tecnología Guía del participante. Lima: CONCYTEC; 2002

3. Ishiyama Cervantes, Raúl. Enseñar Aprender la ciencia como investigación. Lima: Universidad Peruana Cayetano Heredia; 1995

4. Ishiyama Cervantes, Raúl. Mecanismos de producción de la creatividad en ciencia y tecnología. En: Ciencia y Tecnología para el desarrollo. Lima: CONCYTEC; 1989. pp.179-184.

5. Hallasi Roselló, Dilma D. e Ishiyama Cervantes, Raúl. Viviendo la educación con salud. En: 4to Encuentro Internacional sobre Educación Productiva. Huacho: Colegio de Ciencias; 2002. pp. 126 - 129.

6. Ishiyama Cervantes, Raúl. Pautas para la publicación de artículos científicos. Lima: Universidad Peruana Cayetano Heredia; 2003. (Edición virtual) Se encuentra en:

http://www-upch.edu.pe/upchvi/lnvestigacion/upch.htm http://www.upch.edu.pe/upchvi/lnvestigacion/pautas/upch.html

7. Gelb, Michael J. La inteligencia genial Leonardo da Vinci. Bogotá: Editorial Norma; 1998.

8. Thorpe, Scott. Cómo pensar como Einstein. Bogotá: Norma; 2000. 